\title{
The 2014-2015 National Impact of the 2014 American Academy of Pediatrics Guidance for Respiratory Syncytial Virus Immunoprophylaxis on Preterm Infants Born in the United States
}

\author{
${ }^{1}$ Watson Health Value Based Care, Truven Health Analytics, an IBM \\ Company, Cambridge, Massachusetts \\ 2 Pediatric Infectious Diseases, Children's Medical Center, NYU \\ Winthrop, Mineola, New York \\ ${ }^{3}$ Department of Pediatric Infectious Disease, Driscoll Children's \\ Hospital, Corpus Christi, Texas \\ ${ }^{4}$ Division of Neonatal Medicine, Loma Linda University Children's \\ Hospital, Linda Loma, California \\ 5 Wade Outcomes Research and Consulting, Salt Lake City, Utah \\ ${ }^{6}$ Health Economics and Outcomes Research, AstraZeneca, \\ Gaithersburg, Maryland
}

Amanda M. Kong, $\mathrm{MPH}^{1} \quad$ Leonard R. Krilov, $\mathrm{MD}^{2} \quad$ Jaime Fergie, $\mathrm{MD}^{3} \quad$ Mitchell Goldstein, $\mathrm{MD}^{4}$ David Diakun, BS ${ }^{1}$ Sally W. Wade, MPH $^{5}$ Melissa Pavilack, PharmD ${ }^{6} \quad$ Kimmie K. McLaurin, MS 6

\author{
Address for correspondence Jaime Fergie, MD, Driscoll Children's \\ Hospital, 3533 S Alameda Street, Corpus Christi, TX 78411 \\ (e-mail: Jaime.Fergie@dchstx.org).
}

Am J Perinatol 2018;35:192-200.

\section{Abstract \\ Keywords \\ - respiratory syncytial virus \\ - immunoprophylaxis \\ - hospitalization rates \\ - palivizumab}

Objective This article aims to compare respiratory syncytial virus (RSV) immunoprophylaxis (IP) use and RSV hospitalization rates (RSVH) in preterm and full-term infants without chronic lung disease of prematurity or congenital heart disease before and after the recommendation against RSV IP use in preterm infants born at 29 to 34 weeks' gestational age (WGA).

Study Design Infants in commercial and Medicaid claims databases were followed from birth through first year to assess RSV IP and RSVH, as a function of infant's age and WGA. RSV IP was based on pharmacy or outpatient medical claims for palivizumab. RSVH was based on inpatient medical claims with a diagnosis of RSV.

Results Commercial and Medicaid infants 29 to 34 wGA represented 2.9 to $3.5 \%$ of all births. RSV IP use in infants 29 to 34 wGA decreased 62 to $95 \%(p<0.01)$ in the 2014-2015 season relative to the 2013-2014 season. Compared with the 2013-2014 season, RSVH increased by 2.7 -fold $(p=0.02)$ and 1.4-fold $(p=0.03)$ for infants aged $<3$ months and 29 to 34 wGA in the 2014-2015 season with commercial and Medicaid insurance, respectively. In the 2014-2015 season, RSVH for infants 29 to 34 wGA were two to seven times higher than full-term infants without high-risk conditions.

Conclusion Following the 2014 RSV IP guidance change, RSV IP use declined and RSVH increased among infants born at 29 to 34 wGA and aged $<3$ months.
Respiratory syncytial virus (RSV) is an infectious agent that causes annual epidemics of serious lower respiratory disease and is a frequent reason for hospitalization among young children in the United States and around the world. ${ }^{1,2}$ Certain

received

June 6, 2017

accepted after revision

July 26, 2017

published online

September 7, 2017 $10.1055 / \mathrm{s}-0037-1606352$ ISSN $0735-1631$. groups of infants are at increased risk for severe RSV disease and related hospitalizations, including preterm infants and infants with chronic lung disease of prematurity (CLDP, previously referred to as bronchopulmonary dysplasia

Copyright $\odot 2018$ by Thieme Medical Publishers, Inc., 333 Seventh Avenue, New York, NY 10001, USA.

License terms

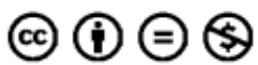


[BPD]) or hemodynamically significant congenital heart disease (CHD). ${ }^{3}$ Multiple studies have shown that RSV hospitalization risk increases as gestational age decreases ${ }^{4}$ and is greater among infants at younger chronological ages during the RSV season (November through March). ${ }^{3,5-7}$

In 1998, palivizumab, a monoclonal antibody, was approved for the prevention of severe RSV infection in children with BPD and children born at $\leq 35$ wGA. In 2003, an additional indication was obtained for children with hemodynamically significant CHD. ${ }^{8}$ To date, no other Food and Drug Administration-approved prophylaxis or treatment for severe RSV disease exists. The American Academy of Pediatrics (AAP) Committee on Infectious Diseases (COID) has regularly issued guidance on the use of RSV immunoprophylaxis (IP) in the United States, and recent changes have redefined the recommendations for RSV IP in preterm infants. In 2014, the AAP recommended against the use of RSV IP in all infants born at 29 to 34 wGA unless the infant had an underlying health condition, such as BPD/CLDP or CHD. ${ }^{9}$

The national impact of the 2014 policy change on the utilization of RSV IP and rates of RSV hospitalizations among preterm infants in the United States has been assessed in few studies to date. ${ }^{10-13}$ None of these studies evaluated national RSV hospitalization rates in the affected infant population. To address this gap in the literature, we used data from two large national cohorts of commercially and Medicaid-insured infants to test the hypothesis that RSV IP decreased and RSV hospitalization rates increased in infants born at 29 to 34 wGA after the guidance change. This study compared RSV IP utilization and RSV hospitalization rates among preterm infants 29 to 36 wGA during the 2014-2015 season with rates in the 2013-2014 season and the combined 20102014 seasons. RSV IP and RSV hospitalization rates for fullterm infants were also captured to identify seasonal variations that may impact all infants.

\section{Methods}

\section{Data Sources}

This analysis utilized the Truven Health MarketScan Commercial and Multi-State Medicaid health insurance administrative claims databases. These databases comprise health care claims and enrollment information for enrollees from self-insured employers and commercial health plans, as well as enrollees in Medicaid from several geographically diverse states. Between 1995 and 2015 overall, approximately 132 million enrollees were included in the commercial database and approximately 40 million enrollees were included in the Medicaid database. Inpatient and outpatient medical claims and outpatient pharmacy claims are included in the databases. These databases have been used in more than 1,000 peer-reviewed articles, ${ }^{14}$ including several manuscripts related to RSV. ${ }^{15-18}$ The data were previously collected and statistically de-identified, and are compliant with the conditions set forth in Sections 164.514(a)-(b)(1)ii of the Health Insurance Portability and Accountability Act of 1996 Privacy Rule; therefore, approval from an institutional review board was not required.

\section{Patient Selection and Follow-up}

Infants born from July 1, 2009, through June 30, 2015, were identified in inpatient claims using ICD-9-CM diagnosis codes and diagnosis-related group (DRG) codes. To capture complete outcomes data for the 2010-2011 RSV season, infants born in the 2009-2010 season were included. Infants who were discharged alive from their birth hospitalization were classified into risk cohorts using diagnoses, DRGs, procedures, and medications appearing in their medical and pharmacy claims during the first 90 days of life (-Supplementary Table 1, available in the online version). The following classification was applied hierarchically to generate mutually exclusive cohorts: infants with rare, complex medical conditions (e.g., infants with evidence of cystic fibrosis, immunodeficiency, congenital anomalies of the respiratory system, neuromuscular, immunological, or genetic conditions, or organ transplants); infants with CLDP; infants with CHD; infants born at $<29$ wGA, 29 to 30 wGA, 31 to $32 \mathrm{wGA}, 33$ to $34 \mathrm{wGA}, 35$ to $36 \mathrm{wGA}$; infants born at $>36$ wGA (full-term) with major health problems; infants born at $>36$ wGA without major health problems; infants with wGA unknown but with codes indicating preterm birth; and infants with wGA unknown and no information regarding preterm or full-term birth. This analysis focused on preterm infants (born 29-30 wGA, 31-32 wGA, and 33-34 wGA), as these infants were most affected by the 2014 change in RSV IP recommendations, with comparisons to preterm infants 35 to 36 wGA and full-term infants without major health problems, who were not affected by the change.

Infants were followed up from birth through the end of the first year of life, death, or end of continuous enrollment in the database, whichever came first. Although there is geographical and annual variability in the timing of RSV circulation in the United States, the RSV season was defined in accordance with the national annual average as occurring between November 1 and March 31, based on data collected and published by the Centers for Disease Control and Prevention. ${ }^{19}$ Rates were calculated based on events per 100 infant-seasons, with the infant-season denominator calculated from the total days contributed during the RSV season divided by 151 days, which reflects the total number of days during the RSV season. Rates were determined for the entire first year of life and for prespecified age intervals $(<3$ months, 3 to $<6$ months, and 6-12 months old). Because infants were followed up for up to one year, they may have contributed person-time to more than one RSV season. For example, an infant born in January 2010 would contribute person-time to the 2010-2011 season as well as to the 20112012 season. Similarly, an infant could contribute time to multiple age groups depending on date of birth relative to the RSV season. However, comparative analyses between seasons were limited to infants $<6$ months old; therefore, each infant could only contribute to one RSV season.

\section{Study Variables}

The primary variables of interest were RSV IP receipt and RSVcoded hospitalizations occurring during the RSV seasons in 2010-2014 (average pre-recommendation), 2013-2014 
(season before recommendation), and 2014-2015 (season after recommendation). RSV IP receipt was identified using outpatient medical and pharmacy claims with palivizumabspecific drug codes (National Drug Code) or administration codes (Current Procedural Terminology or Healthcare Common Procedure Coding System). RSV IP use was analyzed as the proportion of infants with evidence of at least one claim for palivizumab. Inpatient doses are not captured in the databases. RSV-coded hospitalizations were identified using inpatient claims for an ICD-9-CM diagnosis of 079.6, 466.11, or 480.1 in any position. RSV hospitalization rates were calculated as the number of RSV hospitalizations per 100 infant-seasons. Hospitalizations occurring $<2$ days after birth hospitalization discharge were not analyzed to exclude nosocomial RSV infections associated with the birth hospitalization.

Because lower respiratory disease caused by RSV is commonly coded as unspecified bronchiolitis without an RSVspecific code, ${ }^{20}$ a secondary outcome of interest was the rate of all-cause bronchiolitis hospitalizations. All-cause bronchiolitis hospitalizations comprised hospitalizations coded as RSV bronchiolitis (ICD-9-CM code 466.11) and hospitalizations coded as unspecified bronchiolitis (ICD-9-CM code 466.19). Hospitalizations for unspecified bronchiolitis were included only in the absence of diagnosis codes for RSV, influenza, bacterial pneumonia, or other viral pathogens within a 3-day window.

\section{Statistical Analyses}

Data for the commercial and Medicaid populations were analyzed separately. In each population, chi-squared tests compared the proportion of infants with RSV IP during the 2013-2014 and 2014-2015 seasons. To evaluate changes in hospitalization rates from the 2013-2014 season to the 2014-2015 season, generalized linear regression models with Poisson error distribution, log link, and log offset for exposure time were fit. The exposures were wGA, season, sex, and interaction of wGA and season. The models included only infants $<6$ months old, and therefore infants could only contribute to one RSV season. In a sensitivity analysis, models were fit comparing RSV hospitalization rates between the averages of RSV season 2010-2014 and the rate in the 2014-2015 RSV season. $p$-Values $<0.05$ were considered statistically significant. To address seasonal variation in RSV circulation and severity, crude rate ratios (RR) were calculated to compare RSV hospitalization rates of preterm infants to those of full-term infants by season. This approach assumes that variations in RSV hospitalization rates in healthy full-term infants reflect differential seasonal severity, as healthy full-term infants are not indicated for RSV IP.

\section{Results}

\section{Patient Sample}

There were 1,535,884 commercially insured and 1,698,146 Medicaid-insured infants who were born from July 1, 2009, through June 30, 2015, and were discharged alive from birth hospitalization. Of the infants identified, $77.2 \%$ commercially insured ( $n=1,186,080)$ and 79.5\% Medicaid-insured infants ( $n=1,350,354)$ were classified into one of the preterm with identifiable wGA $(<29-36$ wGA) cohorts or one of the full-term cohorts (with or without major health problems; -Supplementary Table 2, available in the online version). The majority of these infants were born full-term without major health problems ( $78.1 \%$ of commercially insured and $80.2 \%$ of Medicaid-insured infants), while $3.8 \%$ of commercially insured infants and $4.4 \%$ of Medicaid-insured infants were born at 29 to 34 wGA. Full study attrition and available demographic information are shown in -Supplementary Tables 3 and $\mathbf{4}$, available in the online version. In the commercially insured sample, 44,698 preterm infants born at 29 to 34 wGA contributed 30,851 infant-seasons of follow-up and 926,483 fullterm infants with no major health problems contributed 693,397 infant-seasons. In the Medicaid sample, 59,356 preterm infants born at 29 to 34 wGA contributed 43,495 infantseasons of follow-up and 1,082,519 full-term infants with no major health problems contributed 852,337 infant-seasons.

\section{RSV Immunoprophylaxis}

Between the 2013-2014 and 2014-2015 seasons, there were significant declines in the proportion of 29 to 34 wGA infants with at least one dose of RSV IP $(p<0.01)$. These declines ranged from 45 to $95 \%$ across the preterm subgroups (-Table 1). The largest absolute declines in the proportion of infants receiving RSV IP occurred among infants born at earlier wGA (e.g., 29-30 wGA) and with chronological age $<6$ months during the RSV season. RSV IP rates for preterm infants born at 35 to 36 wGA and full-term infants were $<1 \%$ in both the 2013-2014 and 2014-2015 RSV seasons. Average RSV IP rates in the 2013-2014 season were similar to those observed during all seasons from 2010 to 2014 .

\section{RSV Hospitalizations}

From July 1, 2010, through June 30, 2015, we identified 6,563 RSV hospitalizations in the commercial database and 13,312 RSV hospitalizations in the Medicaid database among preterm infants 29 to 34 wGA and full-term infants. In both study populations, approximately $88 \%$ of RSV hospitalizations occurred during the RSV season. Additionally, approximately $75 \%$ of RSV hospitalizations among preterm infants 29 to 34 wGA and full-term infants occurred in infants $<6$ months old, and approximately 50\% occurred among infants $<3$ months old.

In the commercially insured population, RSV hospitalization rates ranged from 0.3 hospitalizations per 100 infantseasons (95\% confidence interval [CI], 0.2-0.3) among fullterm infants 6 to 12 months old in the 2013-2014 season to 6.3 hospitalizations per 100 infant-seasons (95\% CI, 2.1-14.8) among preterm infants 29 to 30 wGA who were $<3$ months old in the 2014-2015 RSV season (-Fig. 1A). In the Medicaidinsured population, RSV hospitalization rates ranged from 0.5 hospitalizations per 100 infant-seasons (95\% CI, 0.4-0.5) among full-term infants 6 to 12 months old in the 2014-2015 season to 11.7 hospitalizations per 100 infant-seasons (95\% CI, 7.0-18.3) among preterm infants 29 to 30 wGA who were $<3$ months old in the 2014-2015 RSV season (-Fig. 1B). 
Table 1 RSV immunoprophylaxis use among commercially insured and Medicaid-insured infants by risk cohort

\begin{tabular}{|c|c|c|c|c|c|c|c|c|c|c|c|c|c|c|}
\hline \multirow[b]{3}{*}{ Age and risk cohorts } & \multicolumn{7}{|c|}{ Commercial } & \multicolumn{7}{|c|}{ Medicaid } \\
\hline & \multicolumn{3}{|c|}{$2013-2014^{a}$} & \multicolumn{3}{|c|}{$2014-2015^{a}$} & \multirow{2}{*}{$\begin{array}{l}\text { Absolute } \\
\text { decrease } \\
\text { (\% decrease) }\end{array}$} & \multicolumn{3}{|c|}{$2013-2014^{a}$} & \multicolumn{3}{|c|}{$2014-2015^{a}$} & \multirow{2}{*}{$\begin{array}{l}\text { Absolute } \\
\text { decrease } \\
\text { (\% decrease }\end{array}$} \\
\hline & $\begin{array}{l}\text { No. of } \\
\text { infants }\end{array}$ & $\begin{array}{l}\text { No. w/ } \\
\text { RSV IP }\end{array}$ & $\begin{array}{l}\text { \% RSV } \\
\text { IP use }\end{array}$ & $\begin{array}{l}\text { No. of } \\
\text { infants }\end{array}$ & $\begin{array}{l}\text { No. w/ } \\
\text { RSV IP }\end{array}$ & $\begin{array}{l}\text { \% RSV } \\
\text { IP use }\end{array}$ & & $\begin{array}{l}\text { No. of } \\
\text { infants }\end{array}$ & $\begin{array}{l}\text { No. w/ } \\
\text { RSV IP }\end{array}$ & $\begin{array}{l}\text { \% RSV } \\
\text { IP use }\end{array}$ & $\begin{array}{l}\text { No. of } \\
\text { infants }\end{array}$ & $\begin{array}{l}\text { No. w/ } \\
\text { RSV IP }\end{array}$ & $\begin{array}{l}\text { \% RSV } \\
\text { IP use }\end{array}$ & \\
\hline \multicolumn{15}{|l|}{$<3$ months $^{\mathrm{b}}$} \\
\hline $29-30$ wGA & 418 & 136 & $32.5 \%$ & 388 & 29 & $7.5 \%$ & $25 \%(77 \%)$ & 816 & 239 & $29.3 \%$ & 679 & 32 & $4.7 \%$ & $25 \%(84 \%)$ \\
\hline $31-32$ wGA & 947 & 293 & $30.9 \%$ & 809 & 33 & $4.1 \%$ & $27 \%(87 \%)$ & 1,508 & 451 & $29.9 \%$ & 1,473 & 25 & $1.7 \%$ & $28 \%(94 \%)$ \\
\hline $33-34$ wGA & 2,530 & 519 & $20.5 \%$ & 2,359 & 30 & $1.3 \%$ & $19 \%(94 \%)$ & 4,052 & 675 & $16.7 \%$ & 3,676 & 34 & $0.9 \%$ & $16 \%(95 \%)$ \\
\hline \multicolumn{15}{|l|}{3 to $<6$ months $^{b}$} \\
\hline 29-30 wGA & 470 & 254 & $54.0 \%$ & 521 & 97 & $18.6 \%$ & $35 \%(66 \%)$ & 1,051 & 510 & $48.5 \%$ & 940 & 91 & $9.7 \%$ & $39 \%(80 \%)$ \\
\hline $31-32$ wGA & 953 & 294 & $30.8 \%$ & 980 & 77 & $7.9 \%$ & $23 \%(75 \%)$ & 1,818 & 454 & $25.0 \%$ & 1,724 & 46 & $2.7 \%$ & $22 \%(89 \%)$ \\
\hline $33-34$ wGA & 2,590 & 299 & $11.5 \%$ & 2,677 & 27 & $1.0 \%$ & $11 \%(91 \%)$ & 4,374 & 318 & $7.3 \%$ & 4,118 & 66 & $1.6 \%$ & $6 \%(78 \%)$ \\
\hline \multicolumn{15}{|l|}{6 to $<12$ months $^{\mathrm{b}}$} \\
\hline 29-30 wGA & 695 & 190 & $27.3 \%$ & 688 & 104 & $15.1 \%$ & $12 \%(45 \%)$ & 1,322 & 358 & $27.1 \%$ & 1,262 & 100 & $7.9 \%$ & $19 \%(71 \%)$ \\
\hline $31-32$ wGA & 1,351 & 152 & $11.3 \%$ & 1,413 & 60 & $4.2 \%$ & $7 \%(62 \%)$ & 2,381 & 266 & $11.2 \%$ & 2,187 & 36 & $1.6 \%$ & $10 \%(86 \%)$ \\
\hline $33-34$ wGA & 3,424 & 110 & $3.2 \%$ & 3,549 & 29 & $0.8 \%$ & $2 \%(75 \%)$ & 5,665 & 96 & $1.7 \%$ & 5,519 & 31 & $0.6 \%$ & $1 \%(65 \%)$ \\
\hline
\end{tabular}

Abbreviations: RSV IP, respiratory syncytial virus immunoprophylaxis; wGA, weeks gestational age.

anfants can appear in multiple seasons.

${ }^{\mathrm{b}} \mathrm{Age}$ is captured at any point in the RSV season. Infants may appear in multiple age categories.

The 2014-2015 RSV hospitalization rate for commercially insured infants born at 29 to $34 \mathrm{wGA}$ and $<3$ months old was 2.65 times the rate during the 2013-2014 season $(p=0.0184)$. Among Medicaid-insured infants, the RSV hospitalization rate was 1.41 times higher $(p=0.0313)$ for that infant group in multivariable models ( $\mathbf{- T a b l e ~ 2 )}$ ) Among infants born at 29 to $30 \mathrm{wGA}, 31$ to $32 \mathrm{wGA}$, and 33 to 34 wGA and infants $<3$ months old, there was a consistent trend toward increased risk for RSV hospitalization in the 2014-2015 season, although the increase was not always statistically significant. Among full-term infants, RSV hospitalization rates were similar for the 2013-2014 and 2014-2015 RSV seasons. In comparison to the average rate for seasons from 2010 through 2014, RSV hospitalization rates in the 2014-2015 RSV season were significantly higher for infants born at 29 to $34 \mathrm{wGA}$ and $<3$ months of age (-Supplementary Table 5, available in the online version). For infants 3 to $<6$ months old, there were inconsistent differences in RSV hospitalization rates in 20142015 relative to prior seasons across commercial and Medicaid gestational age subgroups. There was a statistically significant decrease in RSV hospitalization rates for fullterm infants from the 2010-2014 seasons to the 2014-2015 season.

Analyzing the ratio of RSV hospitalization rates between preterm groups and full-term infants within an individual season, RRs among infants at $<6$ months of age were greater in 2014-2015 relative to prior seasons (-Fig. 2A, B). For example, among infants born at 29 to $30 \mathrm{wGA}$ and $<3$ months old, RRs were 1.9 and 2.6 for the commercially and Medicaidinsured infants, respectively, in the combined 2010-2014 seasons versus 4.8 and 6.2 in the 2014-2015 season.

\section{All-Cause Bronchiolitis Hospitalizations}

Rates for all-cause bronchiolitis hospitalizations are presented in -Supplementary Fig. 1, available in the online version. Results were consistent with the results for RSV hospitalizations. Compared with the 2013-2014 season using multivariable models, hospitalization rates were significantly higher in the 2014-2015 season for commercially and Medicaid-insured infants 29 to 34 wGA and $<3$ months old (-Table 2). There were no significant differences among full-term infants.

\section{Discussion}

This analysis of two large U.S. claims databases showed a significant decrease in the proportion of infants receiving RSV IP following the AAP guidance change recommending against RSV IP among infants born at 29 to $34 \mathrm{wGA}$ without CLDP, CHD, or other conditions. Concurrently, the RSV hospitalization rate for 29 to 34 wGA infants $<3$ months old during the RSV season increased significantly, while no increase was observed among full-term infants. The increase in the RSV hospitalization rate among 29 to 34 wGA infants $<3$ months old observed between the seasons immediately before and after the guidance change was also observed when comparing the 2014-2015 RSV season to the average of the 2010-2014 RSV seasons, and this increase was consistent in both commercially and Medicaid-insured infants. Although the ecological design of this study cannot ascribe causality between factors, it appears highly likely that the observed increase in RSV hospitalization rates was due to the decrease in RSV IP, given the established efficacy/effectiveness of palivizumab ${ }^{21-23}$ and that the decreased prophylaxis and increased hospitalization rates both occurred among the subset of infants with the greatest risk of severe RSV disease, namely, those $<3$ months of age during the RSV season. $6,7,24,25$

Prior to this study, the contemporary risk of RSV hospitalization among preterm infants recommended for palivizumab was unknown. Some in the medical community have 


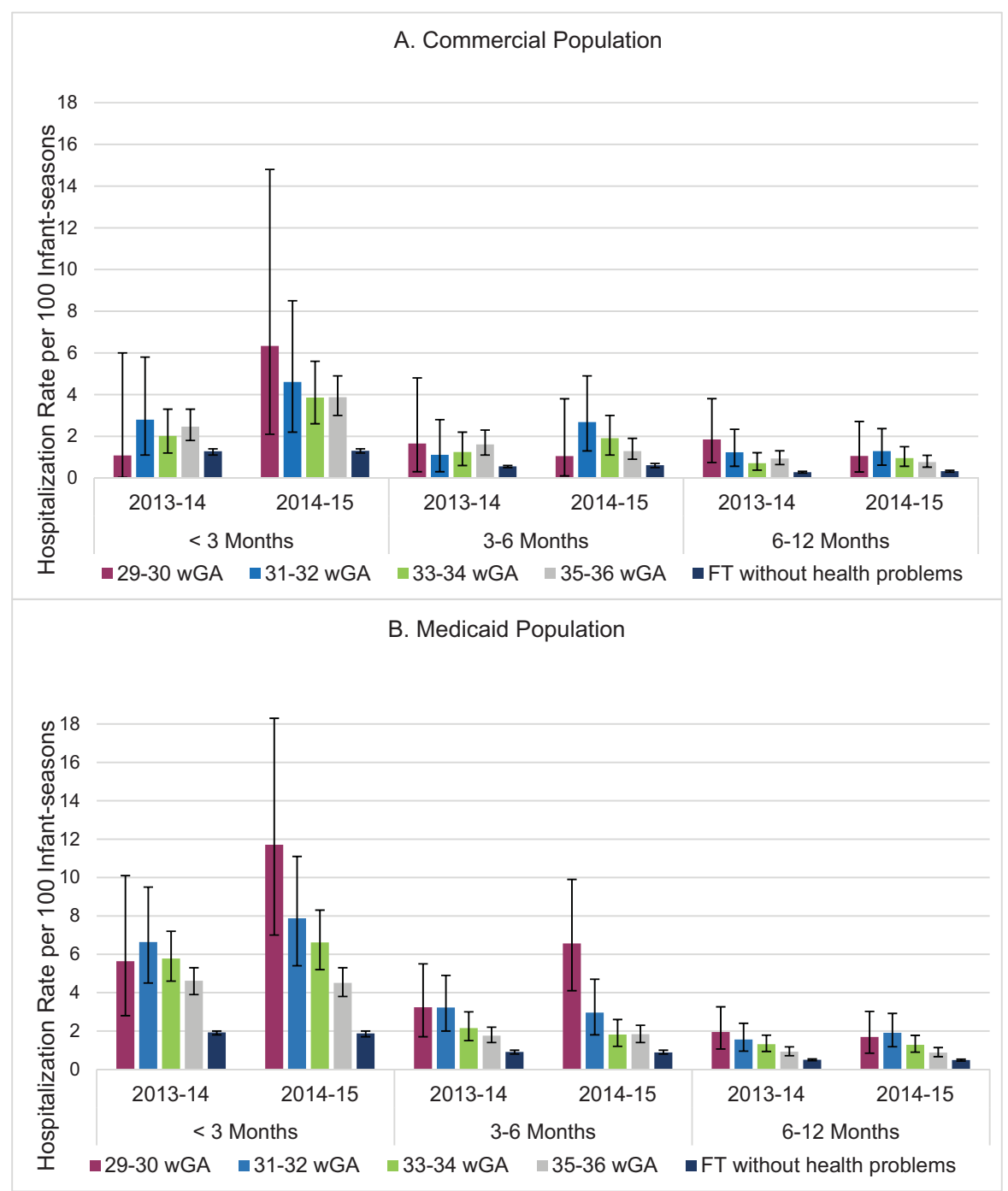

Fig. 1 (A, B) RSV hospitalization rates with 95\% confidence intervals for 2013-2014 and 2014-2015 RSV seasons. FT, full-term; RSV, respiratory syncytial virus; wGA, weeks gestational age.

expressed concern about the AAP policy change, and this has led to a discrepancy in the policy of the AAP and other organizations. ${ }^{21,26}$ The National Perinatal Association recommends that infants born at 28 to $32 \mathrm{wGA}$ and $<6$ months old at the start of the RSV season receive prophylaxis. ${ }^{27}$ The National Perinatal Association also recommends prophylaxis for infants born 32 to $<36 \mathrm{wGA}$ who have significant provider-identified RSV risk factors and are $<6$ months old at the start of the RSV season. ${ }^{27}$

Our analysis provides evidence that the change in AAP RSV IP guidelines likely had a negative impact on premature infants 29 to 34 wGA who were $<3$ months old during the 2014-2015 RSV season. The RSV hospitalization rates observed among 29 to 34 wGA infants in 2014-2015 are similar to rates from studies of RSV hospitalization that were conducted prior to widespread utilization of RSV IP. An analysis of Tennessee Medicaid data from 1989 to 1993 classified infants into mutually exclusive risk categories: BPD, CHD, premature, and other risk factors, and all remaining infants were classified as low risk. ${ }^{3}$ Compared with low-risk infants
$<6$ months old, an increase in risk for RSV/bronchiolitis hospitalization at 0 to 6 months of age was reported for premature infants $\leq 28 \mathrm{wGA}$ (incidence rate ratio [IRR] $=2.1 ; 95 \% \mathrm{CI}, 1.4-3.1)$, premature infants 29 to $32 \mathrm{wGA}$ (IRR $=1.9 ; 95 \% \mathrm{CI}, 1.4-2.4)$, and premature infants 33 to $35 \mathrm{wGA}(\mathrm{IRR}=1.8,95 \% \mathrm{CI}: 1.5-2.1)^{3}$ In another study conducted at the University of Rochester, for infants $<32 \mathrm{wGA}$, it was reported that there was a steady increase in the risk of RSV hospitalization with lower wGA among preterm infants 0 to 12 months without the need for respiratory support after 36 weeks' postconceptual age: $15.0 \%$ for $\leq 26 \mathrm{wGA}, 12.7 \%$ for 27 to $28 \mathrm{wGA}, 10.0 \%$ for 29 to $30 \mathrm{wGA}$, and $6.4 \%$ for 31 to $32 \mathrm{wGA}^{4}$

Additionally, the higher rate of RSV hospitalization that we observed among Medicaid-insured infants compared with commercially insured infants is consistent with previous research indicating that insurance type likely impacts RSV-related facility-based utilization. In that analysis, infants insured through Medicaid had significantly greater incidence of RSV-related emergency room visits as well as 
Table 2 Rate ratios for RSV hospitalization and all-cause bronchiolitis hospitalization in the 2014-2015 RSV season compared with the 2013-2014 RSV season

\begin{tabular}{|c|c|c|c|c|c|c|c|c|}
\hline \multirow[b]{3}{*}{ Age and risk cohorts } & \multicolumn{4}{|c|}{$\begin{array}{l}\text { RSV hospitalizations }^{\mathrm{a}} \\
2014-2015 \text { vs. } 2013-2014 \\
\end{array}$} & \multicolumn{4}{|c|}{$\begin{array}{l}\text { All-cause bronchiolitis hospitalizations }{ }^{\mathrm{a}} \\
2014-2015 \text { vs. 2013-2014 }\end{array}$} \\
\hline & \multicolumn{2}{|l|}{ Commercial } & \multicolumn{2}{|l|}{ Medicaid } & \multicolumn{2}{|l|}{ Commercial } & \multicolumn{2}{|l|}{ Medicaid } \\
\hline & RR $(95 \% \mathrm{Cl})$ & $p$-Value & RR $(95 \% \mathrm{Cl})$ & $p$-Value & $\mathrm{RR}(95 \% \mathrm{Cl})$ & $p$-Value & $\mathrm{RR}(95 \% \mathrm{Cl})$ & $p$-Value \\
\hline \multicolumn{9}{|l|}{$<3$ months $^{\mathrm{b}}$} \\
\hline 29-34 wGA & $2.65(1.18-5.97)$ & 0.0184 & $1.41(1.03-1.94)$ & 0.0313 & $2.10(1.27-3.48)$ & 0.0037 & $1.45(1.10-1.90)$ & 0.0087 \\
\hline 29-30 wGA & $5.94(0.69-50.88)$ & 0.1037 & $2.08(0.99-4.38)$ & 0.0529 & $2.98(0.93-9.49)$ & 0.0653 & $2.51(1.29-4.86)$ & 0.0064 \\
\hline $31-32$ wGA & $1.65(0.63-4.34)$ & 0.3094 & $1.18(0.72-1.94)$ & 0.5035 & $2.05(0.91-4.65)$ & 0.0840 & $1.15(0.76-1.74)$ & 0.5099 \\
\hline 33-34 wGA & $1.90(1.03-3.52)$ & 0.0398 & $1.15(0.84-1.57)$ & 0.3931 & $1.52(0.91-2.54)$ & 0.1081 & $1.05(0.80-1.37)$ & 0.7355 \\
\hline 35-36 wGA & $1.57(1.08-2.30)$ & 0.0187 & $0.97(0.77-1.22)$ & 0.8132 & $1.59(1.15-2.22)$ & 0.0055 & $0.91(0.74-1.10)$ & 0.3264 \\
\hline Full term ${ }^{c}$ & $1.02(0.88-1.18)$ & 0.7966 & $0.98(0.88-1.07)$ & 0.6110 & $1.08(0.95-1.22)$ & 0.2483 & $0.95(0.87-1.03)$ & 0.2095 \\
\hline \multicolumn{9}{|l|}{3 to $<6$ months $^{b}$} \\
\hline $29-34$ wGA & $1.34(0.63-2.83)$ & 0.4472 & $1.17(0.82-1.66)$ & 0.3904 & $1.70(0.99-2.91)$ & 0.0527 & $1.35(1.04-1.75)$ & 0.0227 \\
\hline 29-30 wGA & $0.65(0.11-3.86)$ & 0.6318 & $2.04(1.03-4.06)$ & 0.0410 & $1.69(0.49-5.77)$ & 0.4035 & $1.97(1.22-3.18)$ & 0.0056 \\
\hline 31-32 wGA & $2.42(0.76-7.71)$ & 0.1358 & $0.92(0.49-1.71)$ & 0.7924 & $2.21(0.91-5.37)$ & 0.0804 & $1.27(0.78-2.08)$ & 0.3335 \\
\hline 33-34 wGA & $1.53(0.75-3.16)$ & 0.2457 & $0.84(0.51-1.39)$ & 0.5042 & $1.32(0.76-2.29)$ & 0.3219 & $0.98(0.68-1.41)$ & 0.9217 \\
\hline 35-36 wGA & $0.80(0.48-1.33)$ & 0.3872 & $1.03(0.73-1.46)$ & 0.8470 & $0.82(0.54-1.26)$ & 0.3697 & $0.80(0.62-1.04)$ & 0.1028 \\
\hline Full term ${ }^{c}$ & $1.10(0.90-1.35)$ & 0.3561 & $0.97(0.85-1.11)$ & 0.6548 & $1.01(0.86-1.18)$ & 0.9407 & $0.94(0.84-1.05)$ & 0.2832 \\
\hline
\end{tabular}

Abbreviations: $\mathrm{Cl}$, confidence interval; RR, rate ratio; RSV, respiratory syncytial virus; wGA, weeks gestational age.

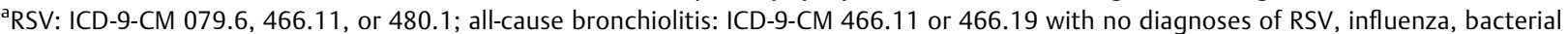
pneumonia, or other viral pathogens.

${ }^{\mathrm{b}}$ Age is captured at any point in the RSV season. Infants may appear in multiple age categories.

'Full-term infants without major health problems.

a greater incidence of RSV-related hospitalizations and intensive care unit admissions, although these differences were not statistically significant. ${ }^{28}$ An analysis of severe lower respiratory tract infections also found that rates among Medicaid-insured children were twice as high as rates among commercially insured children. ${ }^{29}$

Health care utilization related to RSV infection in the 2014-2015 RSV season has been assessed in several data sources, and findings from those studies provide context for our results. Studies conducted at single hospitals by Rajah et al and Blake et al have reported increased RSV morbidity in the 2014-2015 season. ${ }^{11,12}$ Additionally, Anderson et al demonstrated an increased frequency of intensive care unit admission and mechanical ventilation among preterm infants hospitalized with laboratory-confirmed RSV disease during the 2014-2015 RSV season. ${ }^{10}$ Both Rajah et al and Anderson et al noted that infants $<3$ months old were particularly vulnerable. ${ }^{10,11}$ In contrast, Grindeland et al did not observe a significant increase in RSV hospitalizations among all children $<2$ years old in 2014-2015; however, RSV hospitalization rates among preterm infants were not specifically examined, and the study lacked sufficient statistical power to observe the expected differences in subgroups of patients. ${ }^{13,30}$ As a result, failure to demonstrate a difference should not be equated to evidence of a lack of difference. Similarly, Farber examined seasonal RSV and bronchiolitis hospitalization rates among 29 to $<32$ wGA infants in Texas Medicaid plans during 2012-2015 and did not observe a difference across seasons. ${ }^{31}$ However, Texas Medicaid con- tinued to provide RSV prophylaxis to 29 to $<32$ wGA infants in 2014-2015, so no difference would be expected. ${ }^{32}$

Although the impact of the AAP guidance change may be significant to the population of infants 29 to $34 \mathrm{wGA}$, it may be obscured when evaluating the burden of RSV on all infants as a single group. ${ }^{33}$ Because infants 29 to 34 wGA represent approximately $3 \%$ of all births ${ }^{34}$ and not all are $<3$ months old during the RSV season, the negative impact in this small but high-risk population will be undetectable in analyses that combine data for infants of all gestational ages and chronological ages. The need for prophylaxis varies substantially depending on infant characteristics, and it is, therefore, critical for studies that inform prophylaxis recommendations to examine outcomes in individual risk groups and to account for factors such as chronological age during the RSV season.

This analysis has limitations that should be noted. RSV hospitalizations were identified using ICD-9-CM diagnosis codes on inpatient claims. Confirmatory laboratory results were not available, as claims databases typically lack these measures. However, high concordance between RSV diagnosis codes in the emergency room setting and positive RSV tests has been found in a previous study. ${ }^{20}$ Additionally, RSV may be undercoded given that RSV testing is not recommended by the AAP because it does not change the course of treatment. ${ }^{9}$ The increased rates of all-cause bronchiolitis hospitalization in 29 to 34 wGA infants in 2014-2015 demonstrate that there was an overall increase in bronchiolitis hospitalizations, regardless of whether they were 


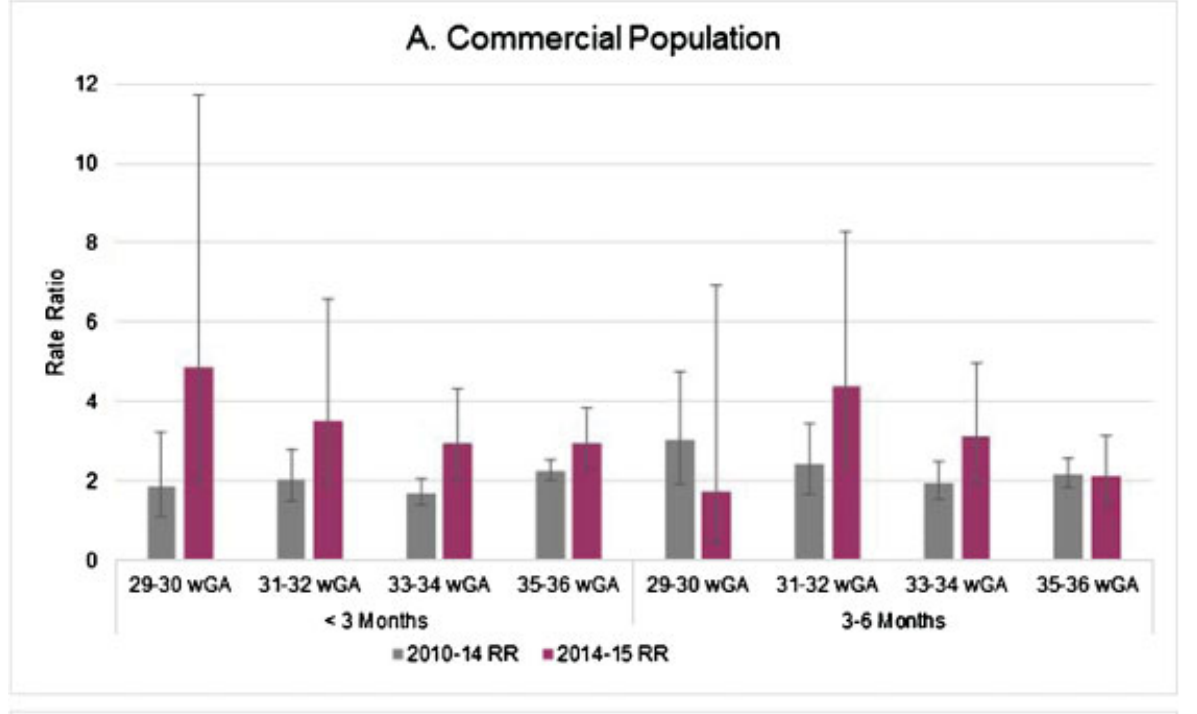

\section{B. Medicaid Population}

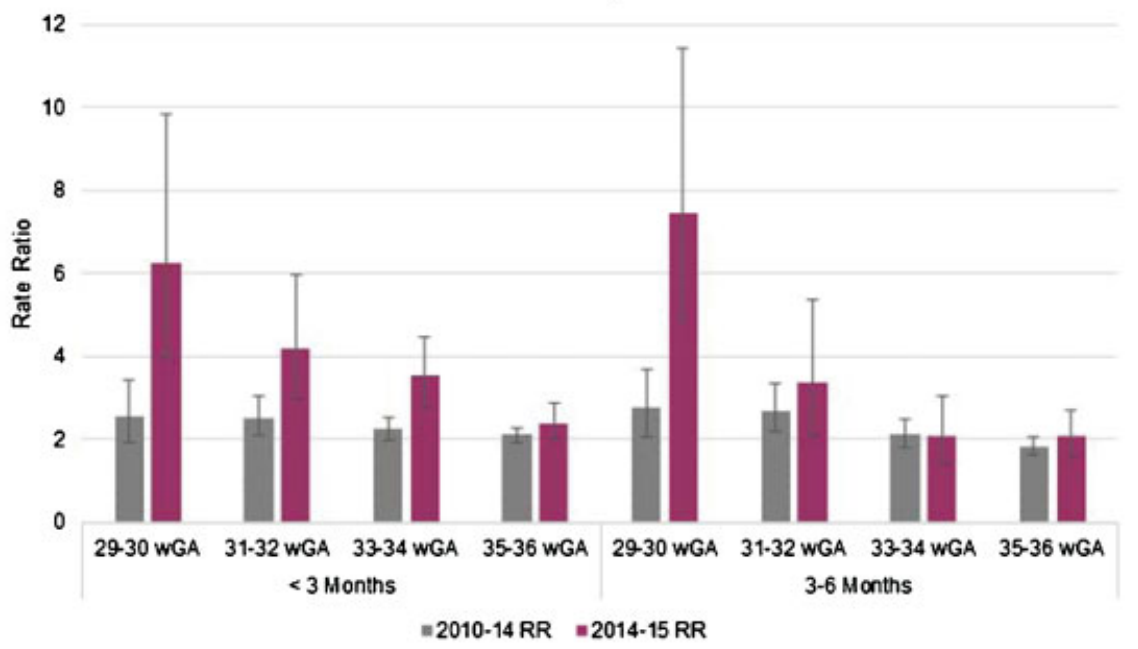

Fig. 2 (A, B) Rate ratios for RSV hospitalization comparing preterm infants to full-term infants by season. RSV, respiratory syncytial virus; wGA, weeks gestational age.

coded as RSV. Utilization of RSV IP among infants $<3$ months old discharged from their birth hospitalization during the RSV season is underestimated because inpatient RSV IP is not recorded in claims databases. Although it may appear counterintuitive that palivizumab utilization was lower among preterm infants $<3$ months relative to those 3 to 6 months of age, this is most likely the result of our inability to capture inpatient administration and the fact that preterm infants are often not discharged home until many weeks following birth. Additionally, our rates likely underestimate the burden of RSV hospitalization under a scenario of no prophylaxis because some infants included in this analysis received outpatient RSV IP despite the changes in recommendations. The burden will likely be increased as compliance with the AAP recommendation increases. Despite using two large databases, there were small numbers of infants in some groups, leading to wide CIs and limiting the statistical power to detect differences between seasons. For this reason, the overall results for 29 to $34 \mathrm{wGA}$ infants are the most robust. Our use of hospitalization rates among non-high-risk fullterm infants as a reference group may not have fully addressed the potential variation in RSV season severity. Race was available only in the Medicaid database and was therefore not included as a variable in any of the multivariable modeling.

In conclusion, this study provides a national estimate of the real-world impact of the AAP's change in recommendations for RSV IP for 29 to 34 wGA infants without CLDP and CHD. Following this recommendation change, the rates of RSV IP declined significantly and the rates of RSV hospitalization increased significantly, particularly among 29 to 34 wGA infants $<3$ months old during the RSV season. These findings are of substantial clinical concern given the potential for further increase in insurer acceptance of and provider compliance with the AAP guidance, and will be reevaluated when data from the 2015-2016 RSV season become available. 


\section{Note}

This work was presented as a poster titled "Impact of 2014 American Academy of Pediatrics Guidance for RSV Immunoprophylaxis" at AMCP Nexus 2016, National Harbor, MD, October 3-6, 2016, and was presented as a platform presentation titled "Impact of 2014 American Academy of Pediatrics Policy for Respiratory Syncytial Virus (RSV) Immunoprophylaxis" at the Pediatric Academic Societies (PAS) 2017 Meeting, San Francisco, CA, May 6-9, 2017.

\section{Conflict of Interest}

Kimmie McLaurin and Melissa Pavilack are employees of AstraZeneca; David Diakun and Amanda M. Kong are employees of Truven Health Analytics; and Sally Wade is a consultant to Truven Health Analytics, which was contracted by AstraZeneca for data analyses and writing support; Leonard Krilov and Mitchell Goldstein have received grant/research support from AstraZeneca/MedImmune; and Jaime Fergie has received grant/research support from MedImmune and was a member of the MedImmune Speakers' Bureau.

\section{Acknowledgments}

This analysis was funded by AstraZeneca. The authors would like to acknowledge Lorena Lopez-Gonzalez, Isabelle Winer, and Greg Lenhart for analytical support. Editorial support was provided by The Lockwood Group, which was in accordance with Good Publication Practice (GPP3) guidelines and funded by AstraZeneca.

\section{References}

1 Stockman LJ, Curns AT, Anderson LJ, Fischer-Langley G. Respiratory syncytial virus-associated hospitalizations among infants and young children in the United States, 1997-2006. Pediatr Infect Dis J 2012;31(01):5-9

2 Nair H, Nokes DJ, Gessner BD, et al. Global burden of acute lower respiratory infections due to respiratory syncytial virus in young children: a systematic review and meta-analysis. Lancet 2010; 375(9725):1545-1555

3 Boyce TG, Mellen BG, Mitchel EF Jr, Wright PF, Griffin MR. Rates of hospitalization for respiratory syncytial virus infection among children in Medicaid. J Pediatr 2000;137(06):865-870

4 Stevens TP, Sinkin RA, Hall CB, Maniscalco WM, McConnochie KM. Respiratory syncytial virus and premature infants born at 32 weeks' gestation or earlier: hospitalization and economic implications of prophylaxis. Arch Pediatr Adolesc Med 2000;154(01):55-61

5 Ambrose CS, Anderson EJ, Simões EA, et al. Respiratory syncytial virus disease in preterm infants in the U.S. born at 32-35 weeks gestation not receiving immunoprophylaxis. Pediatr Infect Dis J 2014;33(06):576-582

6 Winterstein AG, Knox CA, Kubilis P, Hampp C. Appropriateness of age thresholds for respiratory syncytial virus immunoprophylaxis in moderate-preterm infants: a cohort study. JAMA Pediatr 2013; 167(12):1118-1124

7 Mauskopf J, Margulis AV, Samuel M, Lohr KN. Respiratory syncytial virus hospitalizations in healthy preterm infants: systemic review. Pediatr Infect Dis J 2016;35(07):e229-e238

8 Synagis ${ }^{\circledR}$ (Palivizumab) [Prescribing Information]. Gaithersburg, MD: AstraZeneca; 2014
9 American Academy of Pediatrics Committee on Infectious Diseases; American Academy of Pediatrics Bronchiolitis Guidelines Committee. Updated guidance for palivizumab prophylaxis among infants and young children at increased risk of hospitalization for respiratory syncytial virus infection. Pediatrics 2014; 134(02):415-420

10 Anderson EJ, Krilov LR, DeVincenzo JP, et al. SENTINEL1: an observational study of respiratory syncytial virus hospitalizations among U.S. infants born at 29 to 35 weeks' gestational age not receiving immunoprophylaxis. Am J Perinatol 2017;34(01): 51-61

11 Rajah B, Sánchez PJ, Garcia-Maurino C, Leber A, Ramilo O, Mejias A. Impact of the updated guidance for palivizumab prophylaxis against respiratory syncytial virus infection: a single center experience. J Pediatr 2017;181:183-188.e1

12 Blake SM, Tanaka D, Bendz LM, Staebler S, Brandon D. Evaluation of the financial and health burden of infants at risk for respiratory syncytial virus. Adv Neonatal Care 2017;17(04):292-298

13 Grindeland CJ, Mauriello CT, Leedahl DD, Richter LM, Meyer AC. Association between updated guideline-based palivizumab administration and hospitalizations for respiratory syncytial virus infections. Pediatr Infect Dis J 2016;35(07):728-732

14 Truven Health Analytics. Marketscan studies: abbreviated bibliography. Available at: https://truvenhealth.com/Portals/0/Assets/ Life-Sciences/Bibliographies/2016-Truven-Health-MarketScanBibliography.pdf. Accessed March 29, 2017

15 Palmer L, Hall CB, Katkin JP, et al. Respiratory outcomes, utilization and costs 12 months following a respiratory syncytial virus diagnosis among commercially insured late-preterm infants. Curr Med Res Opin 2011;27(02):403-412

16 Palmer L, Hall CB, Katkin JP, et al. Healthcare costs within a year of respiratory syncytial virus among Medicaid infants. Pediatr Pulmonol 2010;45(08):772-781

17 Krilov LR, Masaquel AS, Weiner LB, Smith DM, Wade SW, Mahadevia PJ. Partial palivizumab prophylaxis and increased risk of hospitalization due to respiratory syncytial virus in a Medicaid population: a retrospective cohort analysis. BMC Pediatr 2014; $14: 261$

18 McLaurin KK, Farr AM, Wade SW, Diakun DR, Stewart DL. Respiratory syncytial virus hospitalization outcomes and costs of full-term and preterm infants. J Perinatol 2016;36(11):990-996

19 Haynes AK, Prill MM, Iwane MK, Gerber SI; Centers for Disease Control and Prevention (CDC). Respiratory syncytial virus-United States, July 2012-June 2014. MMWR Morb Mortal Wkly Rep 2014;63(48): 1133-1136

20 Makari D, Staat MA, Henrickson KJ, Wu X, Ambrose CS. The underrecognized burden of respiratory syncytial virus among infants presenting to US emergency departments. Clin Pediatr (Phila) 2015;54(06):594-597

21 The IMpact-RSV Study Group. Palivizumab, a humanized respiratory syncytial virus monoclonal antibody, reduces hospitalization from respiratory syncytial virus infection in high-risk infants. Pediatrics 1998;102(3, Pt 1):531-537

22 Blanken MO, Rovers MM, Molenaar JM, et al; Dutch RSV Neonatal Network. Respiratory syncytial virus and recurrent wheeze in healthy preterm infants. N Engl J Med 2013;368(19):1791-1799

23 Anderson EJ, Carosone-Link P, Yogev R, Yi J, Simões EAF. Effectiveness of palivizumab in high-risk infants and children: a propensity score weighted regression analysis. Pediatr Infect Dis J 2017;36(08):699-704

24 Hall CB, Weinberg GA, Blumkin AK, et al. Respiratory syncytial virus-associated hospitalizations among children less than 24 months of age. Pediatrics 2013;132(02):e341-e348

25 Simões EA, Anderson EJ, Wu X, Ambrose CS. Effects of chronologic age and young child exposure on respiratory syncytial virus disease among US preterm infants born at 32 to 35 weeks gestation. PLoS One 2016;11(11):e0166226 
200 2014-2015 Impact of the 2014 AAP Guidance in the U.S. Kong et al.

26 Yogev R, Krilov LR, Fergie JE, Weiner LB. Re-evaluating the new Committee on Infectious Diseases recommendations for palivizumab use in premature infants. Pediatr Infect Dis J 2015;34 (09):958-960

27 Goldstein M, Merritt TA, Phillips R, et al. National Perinatal Association 2015 respiratory syncytial virus (RSV) prevention guideline. Neonatology Today 2014;9(11):1-11

28 Franklin JA, Anderson EJ, Wu X, Ambrose CS, Simões EA. Insurance status and the risk of severe respiratory syncytial virus disease in United States preterm infants born at 32-35 weeks gestational age. Open Forum Infect Dis 2016;3(03):ofw163

29 Greenbaum AH, Chen J, Reed C, et al. Hospitalizations for severe lower respiratory tract infections. Pediatrics 2014;134(03):546-554

30 Ambrose CS. Statistical power to detect an association between guideline-based palivizumab administration and hospitalizations for respiratory syncytial virus infections. Pediatr Infect Dis J 2017 ; 36(03):348

31 Farber HJ. Impact of the 2014 American Academy of Pediatrics guidance on respiratory syncytial virus and bronchiolitis hospitalization rates for infants born prematurely. J Pediatr 2017; $185: 250$

32 Texas Medicaid/CHIP Vendor Drug Program Fee-For-Service Medicaid Synagis ${ }^{\circledR}$ Request Form, 2014-2015 Season

33 McLaurin KK, Chatterjee A, Makari D. Modeling the potential impact of the 2014 American Academy of Pediatrics respiratory syncytial virus prophylaxis guidance on preterm infant RSV outcomes. Infect Dis Ther 2015;4(04):503-511

34 Hamilton BE, Martin JA, Osterman MJ, Curtin SC, Matthews TJ. Births: final data for 2014. Natl Vital Stat Rep 2015;64(12): $1-64$ 